\title{
Signal Transducer and Activator of Transcription 3 Staining Method
}

National Cancer Institute

\section{Source}

National Cancer Institute. Signal Transducer and Activator of Transcription 3 Staining

Method. NCl Thesaurus. Code C122872.

An immunohistochemical technique used to detect the presence of signal transducer and activator of transcription 3 in a tissue sample. 